\title{
كلية العلوم الصحية

\section{Development And In Vivo Testing of Smart Nanoparticles for Enhanced Anti-Cancer Activity and Reduced Cardiotoxicity Associated with Tyrosine Kinase Inhibitors Hüseyin C. Yalcin ${ }^{1}$, Hissa Al-Thani², Samar Shurbaji ${ }^{1}$ \\ ${ }^{1}$ Biomedical Research Center, Qatar University, PO Box 2713, Doha, QATAR \\ 2Biomedical Science Department, College of Health Sciences, Qatar University, Doha, Qatar}

Introduction
- Cancer is a growing global problem that is manifested by the uncontrolled division of
abnormal cells in part of the body.
- Chronic Myeloid leukemia (CML) is the most encountered subtype of leukemia among
adults.
- Anti-cancer therapeutics such as Tyrosine Kinase inhibitors (TKIs) for CML had aid in
improving the overall outcomes of patients and increasing their survival rates. However, due
to some encountered toxicity of these drugs especially in the heart, the usage of
nanotechnology to treat the cancer has been raised.
- Zebrafish is a good model for xenotransplantation of human tumor cells in order to develop
a cancer model to study human cancers and testing of the anti-cancer drugs.

\section{Objectives}

To produce smart nanoparticles (PLGA-PEG-PLGA) and define their characteristics.

To generate a zebrafish xenograft model of $\mathrm{CML}$ cancer

To test for the toxicity of TKIs and the generated NPs on normal zebrafish

To determine the efficacy of the generated NPs as effective anti-cancer drug delivery system by testing them on the zebrafish xenograft model.

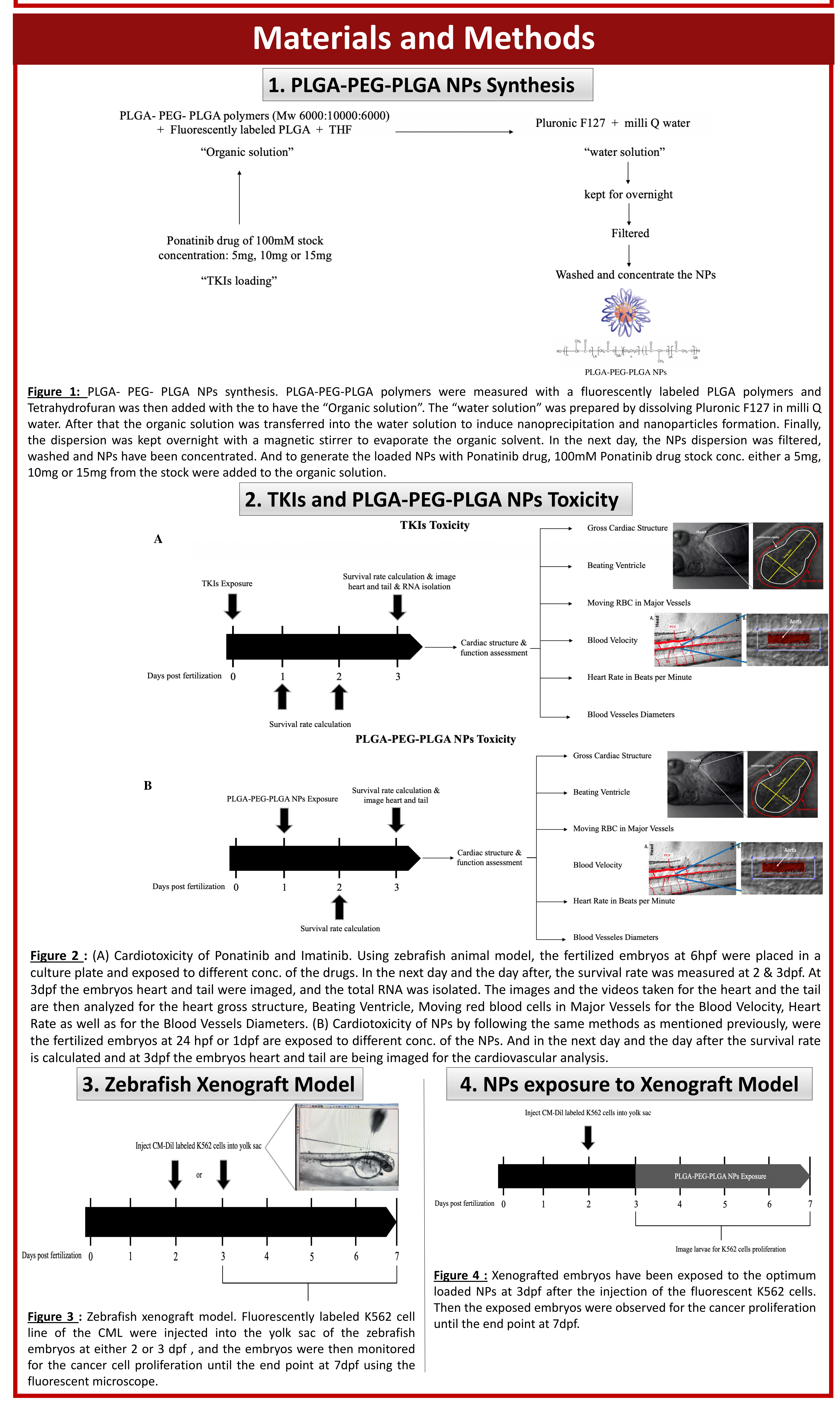

\section{Results and Discussion}

\section{PLGA-PEG-PLGA NPs 3D Structure}

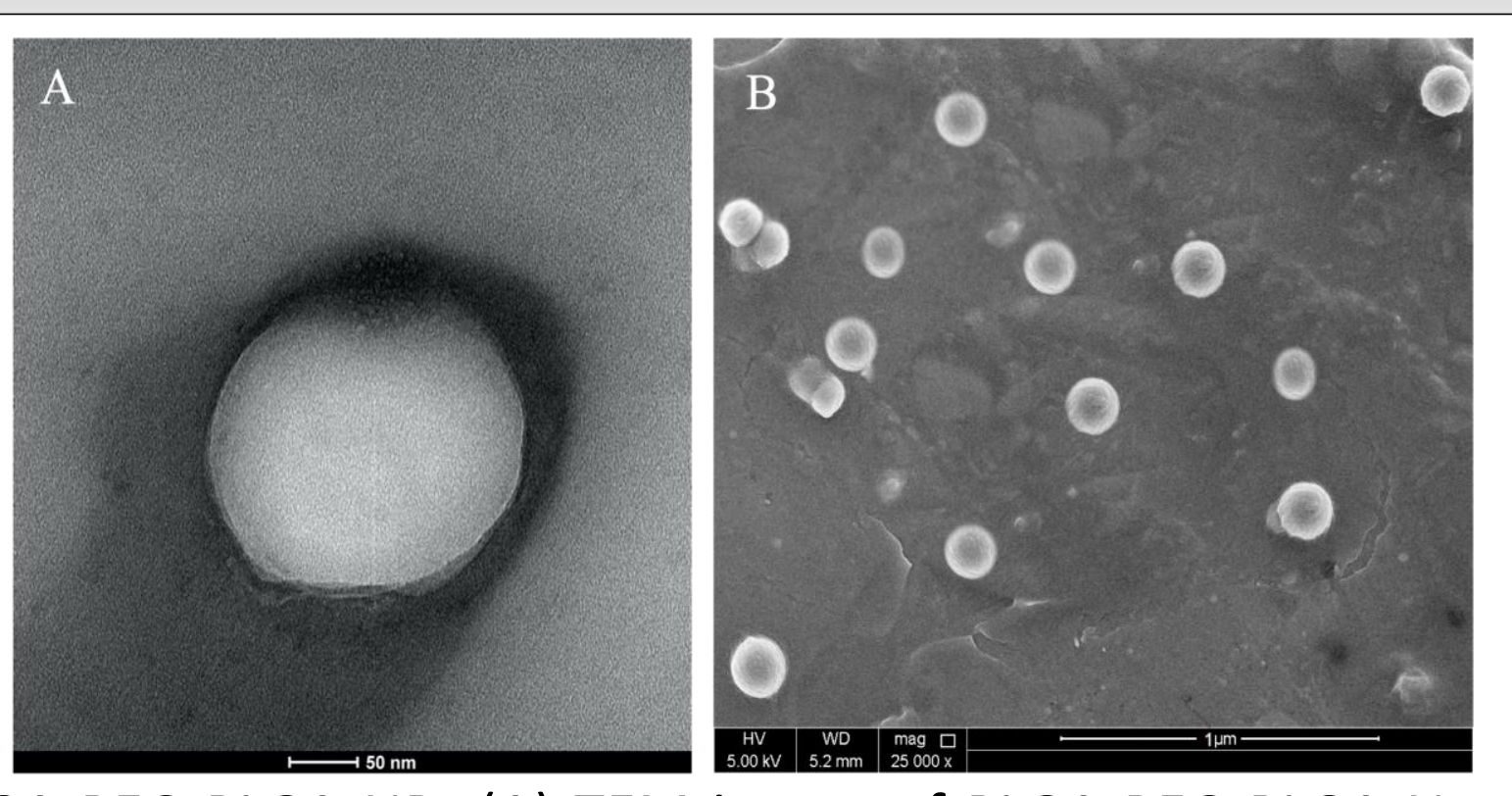

Figure 5 : TEM and SEM micrographs of

\section{Loaded PLGA-PEG-PLGA NPs Toxicity}
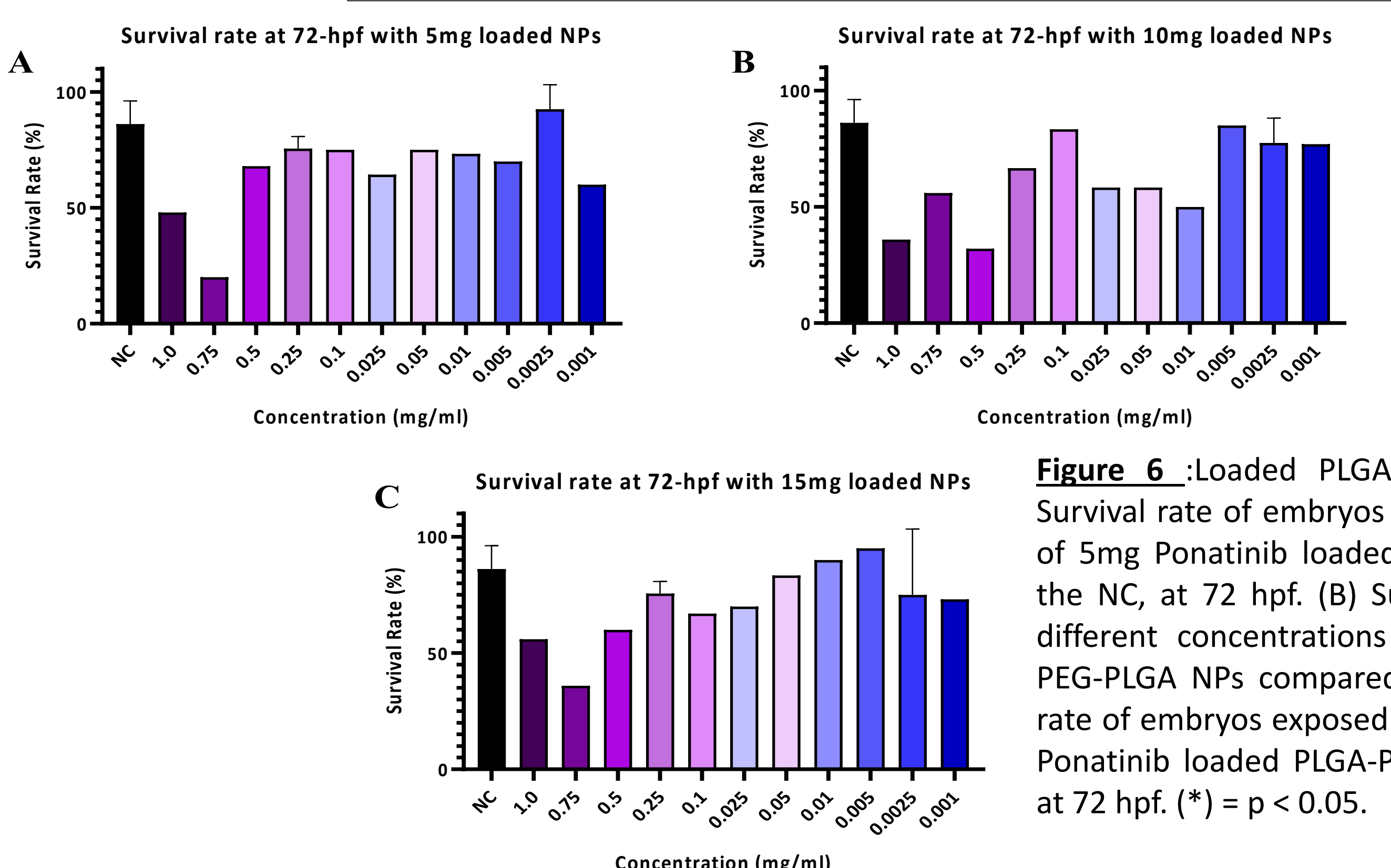

Figure 6 :Loaded PLGA-PEG-PLGA NPS Survival Rate. (A) the NC, at 72 hpf. (B) Survival rate of embryos exposed to diferent concentrations of $10 \mathrm{mg}$ Ponatinib loaded PLGArate of Ponatinib loaded PLGA-PEG-PLGA NPS compared to the NC,
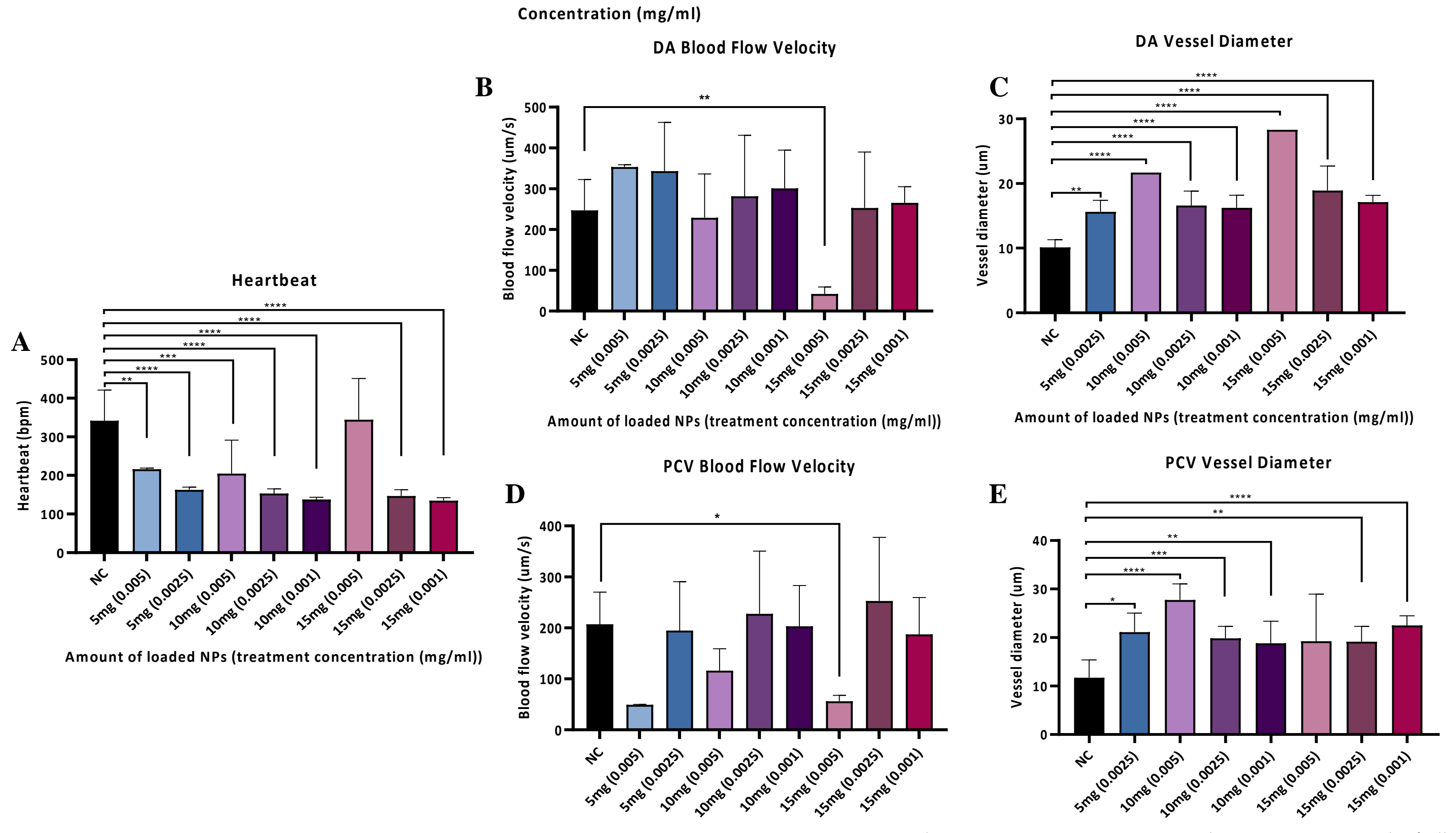

Figure 7: Cardiac function assessment of loaded PLGA-PEG-PLGA NPS.(A) Heartbeat of embryos exposed to different concentrations of loaded PLGA-PEG-PLGA NPs with 5, 10 or 15mg Ponatinib. (B) DA blood flow velocity of embryos exposed to different concentrations of loaded PLGAPEG-PLGA NPs with 5, 10 or $15 \mathrm{mg}$ Ponatinib. (C) DA vessel diameter of embryos exposed to different concentrations of loaded PLGA-PEG-
PLGA NPs with 5,10 or $15 \mathrm{mg}$ Ponatinib. (D) PCV blood flow velocity of embryos exposed to different concentrations of loaded PLGA-PEGPLGA NPS with 5,10 or $15 \mathrm{mg}$ Ponatinib. (E) PCV vessel diameter of embryos exposed to different concentrations of loaded PLGA-PEG-PLGA NPs with 5,10 or $15 \mathrm{mg}$ Ponatinib. $\left.\left({ }^{*}\right)=p<0.05 ;{ }^{* * *}\right)=p<0.001,\left({ }^{* * * *}\right)=p<0.0001$.

\section{Zebrafish Xenograft Model}
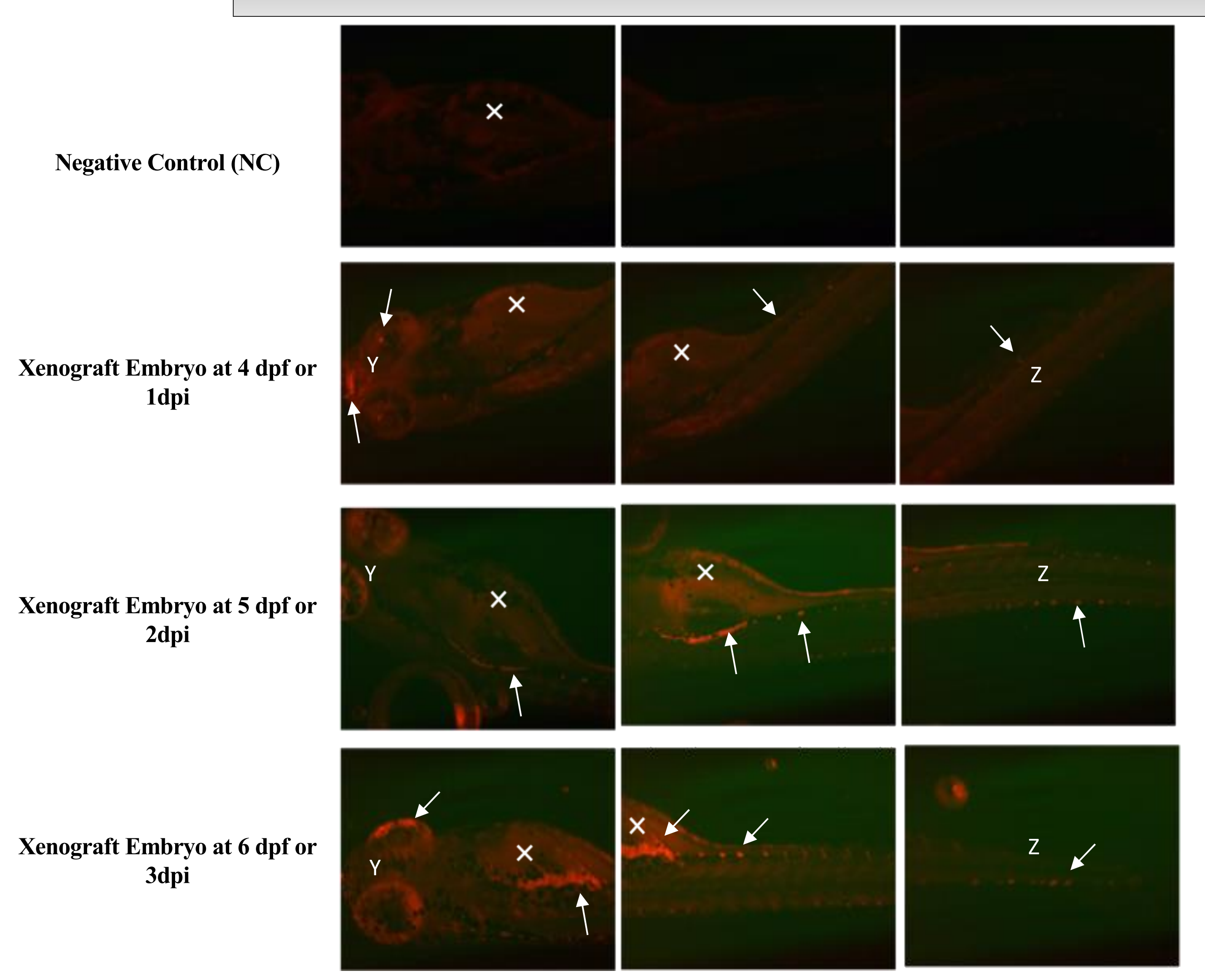

igure 8: Zebrafish Xenograft model injected at $3 \mathrm{dpf}$. Zebrafish screening at $4 \mathrm{dpf}$ to $6 \mathrm{dpf}$ using fluorescent microscopy and investigation of 562 cells proliferation (White solid arrows) through out the animal body (Y: eyes, $X:$ yolk sac, Z: tail) using mCherry fluorescence

\section{CONCLUSIONS}

Zebrafish is a good animal model for xenografting and investigating the cardiotoxicity associated with the anti-cancer drugs such as TKIs.

The lowest concentration of Imatinib and Ponatinib $(2.5 \mu \mathrm{M})$ is the optimum concentration with the least cardiotoxicity and better survival rate.

The concentrations 0.1 and $0.05 \mathrm{mg} / \mathrm{ml}$ of the unloaded NPs are the best with low cardiotoxicity and high survival rate, while $0.001 \mathrm{mg} / \mathrm{ml}$ of the loaded NPs with 10 or $15 \mathrm{mg}$ Ponatinib has shown to be the optimum concentration.

PLGA-PEG-PLGA NPs could be good candidate for CML treatment, but their cellular 